Bull. Austral. Math. Soc.

37D40,37F30, 37F35

Vol. 65 (2002) [199-209]

\title{
HAUSDORFF DIMENSION OF THE LIMIT SET ON A VISIBILITY MANIFOLD
}

\author{
HYUN JUNG KIM
}

\begin{abstract}
In this paper, for a given Fuchsian group $\Gamma$, we prove an upper estimate for the Hausdorff dimension of the radial limit set in the visibility manifold. Further, if $\Gamma$ is a convex cocompact group, we find the exact Hausdorff dimension of the limit set.
\end{abstract}

\section{INTRODUCTION}

Imagine an infinite array of points in hyperbolic space. We consider the distribution of these points at large distances from an observation point $x$. We define the density at the ideal boundary for the array of points viewed from $x$. That is a class of measures on the ideal boundary, which is called the conformal density or Patterson-Sullivan measure. Suppose $H$ is a $n$-dimensional complete simply connected Riemannian manifold without conjugate points and $\Gamma$ is a discrete group of isometries on $H$, which acts on $H$ freely and properly discontinuously. As an infinite array of points, we consider the orbit $\Gamma x$ of $\Gamma$ for a point $x \in H$. The conformal density for $\Gamma x$ was constructed by Patterson in the case where $\operatorname{dim} H=2$ and the sectional curvature of $H$ is constant $-1([7])$. His construction was generalised by Sullivan to the case where the sectional curvature of $H$ is constant -1 in all dimensions ([8]). In [9], Yue performed the same construction when $H$ has a variable negative curvature, and the author proved the existence and some properties of the Patterson-Sullivan measure on a visibility manifold $([6])$.

We define the visibility manifold, following the notations in [2] and [3].

DEFINITION 1.1: $H$ satisfies the visibility axiom if for every point $p \in H$ and every number $\varepsilon>0$, there is $R=R(p, \varepsilon)>0$ such that for any geodesic $\gamma: \mathbb{R} \rightarrow H$ with $d(p, \gamma) \geqslant R, \angle_{p}(\gamma)=\sup \left\{\angle_{p}(\gamma(t), \gamma(s)) \mid t, s \in \mathbb{R}\right\} \leqslant \varepsilon$.

In particular, when we can get the constant $R$ independently of the choice of $p$, we say that $H$ satisfies the uniform visibility axiom.

Received 14th June, 2001

This work was supported by a postdoctoral fellowship from KOSEF.

Copyright Clearance Centre, Inc. Serial-fee code: 0004-9727/02 \$A2.00+0.00. 
In Definition 1.1 the notation $\angle_{p}\left(q_{1} q_{2}\right)$ for $q_{1}$ and $q_{2}$ in $H$ which means the canonical measurement for the angle consisting of two geodesic rays from $p$ to $q_{1}$ and $q_{2}$.

In [1] there are many properties that are equivalent to the visibility axiom in Definition 1.1, and in this paper we use the property below frequently.

For any distinct two points $\eta$ and $\xi$ in $\partial H$, there exists a geodesic line between $\eta$ and $\xi$.

We note that there may be more than one geodesics between $\eta$ and $\xi$. If we have two geodesic between $\eta$ and $\xi$, then the two geodesics bound a flat strip, and in the flat strip, the sectional curvature is 0 . In this paper, we assume the uniform visibility axiom on $H$.

Definition 1.2: Suppose $M$ is a manifold without any conjugate points. If the universal cover $H$ of $M$ satisfies the uniform visibility axiom, we call $M$ a visibility manifold.

Suppose $H$ satisfies the uniform visibility axiom. Let $\partial H$ be the ideal boundary, which is the set of points at infinity for $H$ with the cone topology. Then $H$ is diffeomorphic to an open disc $D^{n}$ and the ideal boundary $\partial H$ of $H$ at infinity is homeomorphic to a sphere $S^{n}$. Let $\Gamma$ be a subgroup of isometries of $H$, that is torsion free, discrete acting on $H$ freely and properly discontinuously in $H$. For any point $x$ in $H$, consider the orbit $\Gamma x$ and its closure $\overline{\Gamma x}$. The limit set of $\Gamma$ is defined by $L(\Gamma)=\overline{\Gamma x} \cap \partial H$. According to Eberlein $[2], L(\Gamma)$ has one point, two points or infinitely many points. From now on, we deal with the case that $L(\Gamma)$ consists of infinitely many points, and call $\Gamma$ the Fuchsian group. Generally, in a 2-dimensional manifold, $\Gamma$ has been called a Fuchsian group, and in higher dimensional manifolds, $\Gamma$ has been called a Kleinian group. Here we call $\Gamma$ Fuchsian group in any dimensional manifold.

We introduce the construction and some properties of the Patterson-Sullivan measure, which were proved in [6] for the visibility manifold.

For positive real number $s$ and two fixed points $x, y$ in $H$, we consider the following Poincaré series

$$
g_{s}(x, y)=\sum_{\gamma \in \Gamma} e^{-s d(x, \gamma y)}
$$

where $d(x, \gamma y)$ is the hyperbolic distance in $H$. Then there is a positive number $\delta(\Gamma)$ such that $g_{s}(x, y)$ diverges for $s<\delta(\Gamma)$ and $g_{s}(x, y)$ converges for $s>\delta(\Gamma)$, that is independent of points $x, y \in H$. 
Define a family of measures

$$
\begin{aligned}
\mu_{x} & =\lim _{s \rightarrow \delta(\Gamma)^{+}} \frac{1}{g_{s}(y, y)} \sum_{\gamma \in \Gamma} e^{-s d(x, \gamma y)} \delta_{\gamma y}, \quad s>\delta(\Gamma), \\
& =\lim _{s \rightarrow \delta(\Gamma)^{+}} \mu_{x}^{s}
\end{aligned}
$$

where $\delta_{\gamma y}$ is the Dirac mass at $\gamma y$. When at $s=\delta(\Gamma) \mu_{x}^{s}$ diverges, $\Gamma$ is of divergence type. Otherwise, $\Gamma$ is of convergence type. When $\Gamma$ is of divergence type, $\mu_{x}$ is concentrated on $L(\Gamma)$. In [6], we proved that for any other point $x, x^{\prime} \in H, \mu_{x^{\prime}}$ and $\mu_{x}$ were absolutely continuous and moreover, the Radon-Nikodym derivative at $\xi \in L(\Gamma)$ was

$$
\frac{d \mu_{x^{\prime}}}{d \mu_{x}}(\xi)=e^{-\delta(\Gamma) \rho_{x . \xi}\left(x^{\prime}\right)},
$$

where $\rho_{x, \xi}\left(x^{\prime}\right)$ is a Busemann function.

For $\gamma \in \Gamma$ we find

$$
\gamma^{*} \mu_{x}=\mu_{\gamma(x)} .
$$

Generally, we call the family $\left\{\mu_{x}\right\}$ of measure on $L(\Gamma)$ satisfying (1.1) and (1.2) a $\delta(\Gamma)$-conformal density or an Patterson-Sullivan measure.

In this paper, we estimate the hyperbolic dimension of the limit set of $L(\Gamma)$. For a hyperbolic manifold of constant curvature -1 , there is a canonical metric on the ideal boundary in the Poincare model and the Hausdorf dimension is exactly $\delta(\Gamma)$ in the case where $\Gamma$ is a convex cocompact group. In a hyperbolic manifold with variable negative curvature, there are many possible equivalent metrics on $\partial H$ (see $[\mathbf{4}, \mathbf{5}, \mathbf{9}]$ ). So the Hausdorff dimension is well defined. Our problem is whether there is natural class of metrics on the visibility manifold. In Section 2, we consider a metric on $\partial H$ which was introduced by Kaimanovich and Hamenstädt for strictly negatively curved manifolds. We show that the metric on $\partial H$ is still well defined on the visibility manifold and we estimate the Hausdorff measure of the radial limit set with respect to this metric. Finally, if $\Gamma$ is a convex cocompact group, we find the exact Hausdorff dimension of limit set $L(\delta)$.

\section{Hausdorff Dimension for Convex Cocompact Group}

Let $(X, d)$ be any metric space and $D \geqslant 0$ be a nonnegative constant. Let $A$ be a subset of $X$. For each $\varepsilon>0$, consider

$$
\mathcal{H}_{\varepsilon}^{D}(A) \equiv \inf \left\{\sum_{j=1}^{\infty} \delta_{j}^{D} \mid A \subset \bigcup_{j} B_{x_{j}}\left(\delta_{j}\right), \delta_{j} \leqslant \varepsilon \text { and } x_{j} \in A\right\},
$$


where the infimum is taken among all coverings of $A$ by balls of radius less than or equal to $\varepsilon$. The limit measure

$$
\mathcal{H}_{d}^{D}(A)=\lim _{\varepsilon \rightarrow 0} \mathcal{H}_{\varepsilon}^{D}(A)
$$

is called the $D$-dimensional Hausdorff measure of $A$. The Hausdorff dimension $H D(A)$ is defined to be

$$
H D(A) \equiv \inf \left\{D \mid \mathcal{H}_{d}^{D}(A)=0\right\} \equiv \sup \left\{D \mid \mathcal{H}_{d}^{D}(A)=\infty\right\}
$$

An easy consequence of this definition is that if $0<\mathcal{H}_{d}^{D}(A)<\infty$ then $H D(A)=D$.

Fix a point $x_{0} \in H$. For any $x$ in $H$ and $d>0$, consider the shadow of the ball $B(x, d)$ from $x_{0}$ to $\partial H$ defined by $O_{x_{0}}(x, d)=\left\{\eta \in \partial H \mid c_{x_{0}, \eta} \cap B(x, d) \neq \emptyset\right\}$, where $c_{x_{0}, \eta}$ is the geodesic ray from $x_{0}$ to $\eta$.

Definition 2.1: $\zeta \in \partial H$ is a radial limit point if for some $c>0$ and $x \in H, \zeta$ belongs to infinitely many shadows $O_{x}(\gamma x, c)$, for $\gamma \in \Gamma$. We denote the radial limit set by $L^{r}(\Gamma)$.

In order to estimate the Hausdorff dimension of the radial limit set, first of all we have to define a metric on $\partial H$. We define a metric on $\partial H$, which was introduced by Kaimanovich [5] and Hamenstädt [4]. Fix a point $x \in H$.

Definition 2.2: For any two points $\eta, \xi \in \partial H$ let $D_{x}(\eta, \xi)$ be the minimum distance from $x$ to a geodesic $c$ from $\xi$ to $\eta$. The geodesic metric is defined for all $\varepsilon>0$ to be

$$
d_{x}^{\varepsilon}(\eta, \xi):=e^{-\varepsilon D_{x}(\eta, \xi)}
$$

In this definition $D_{x}(.,$.$) is well defined because the set of the geodesics between$ two points in $H$, that is the flat part in $H$, has a finite width and the range of the distance from $x$ to $c$ is compact interval. We have to show that the metric $d_{x}^{\varepsilon}$ is well defined.

THEOREM 2.3. There exists $\varepsilon_{0}>0$ such that $d_{x}^{\varepsilon}$ is a metric for all $0<\varepsilon<\varepsilon_{0}$ and $x \in M$.

Proof: By the definition of $d_{x}^{\varepsilon}$, we need to prove only the triangle inequality. Let us choose the three points $\xi_{1}, \xi_{2}, \xi_{3} \in \partial H$ and let $c_{1}, c_{2}, c_{3}$ be any geodesics between $\xi_{1}, \xi_{2}$ and $\xi_{2}, \xi_{3}$ and $\xi_{3}, \xi_{1}$, respectively. We can get a point $p \in c_{3}$ so that $d\left(x, c_{3}\right)=$ $d(x, p)$ and let $R_{0}=d\left(c_{3}, x\right)=d(p, x)$, where $d(.,$.$) is the distance induced from the$ given Riemannian metric. Let $\gamma$ be the geodesic ray from $x$ to $p$ We prove the triangle inequality by the two steps below.

First, we consider the special case in which the geodesic ray from $x$ to $\xi_{2}$ goes through $p$ in the geodesic $c_{3}$. Then $\gamma$ and $c_{3}$ orthogonally meet at $p$, that is, 
$\angle_{p}\left(\xi_{1}, \xi_{2}\right)=\pi / 2$ and $\angle_{p}\left(\xi_{2}, \xi_{3}\right)=\pi / 2$. Since $H$ satisfies the uniformly visibility axiom, we can get a constant $R=R(\pi / 2)$ such that $d\left(c_{1}, p\right) \leqslant R$ and $d\left(c_{2}, p\right) \leqslant R$. So we have $d\left(x, c_{1}\right) \leqslant R_{0}+R$ and $d\left(x, c_{2}\right) \leqslant R_{0}+R$. Choose $\varepsilon_{0}=(\ln 2) / R$. Then for all $\varepsilon<\varepsilon_{0}$,

$$
\begin{aligned}
\exp \left(-\varepsilon d\left(x, c_{3}\right)\right) & =\exp \left(-\varepsilon R_{0}\right) \\
& \leqslant 2 \exp \left(-\varepsilon\left(R_{0}+R\right)\right) \\
& \leqslant \exp \left(-\varepsilon d\left(x, c_{1}\right)\right)+\exp \left(-\varepsilon d\left(x, c_{2}\right)\right) \\
& \leqslant d_{x}^{\varepsilon}\left(\xi_{1}, \xi_{2}\right)+d_{x}^{\varepsilon}\left(\xi_{2}, \xi_{3}\right) .
\end{aligned}
$$

Next, we consider the general case. For a real number $\theta \in[0, \pi]$, define $f(\theta)$ as the minimum distance $d(p, c)$ from $p$ to a geodesic $c$ between $\xi_{2}$ and any point in $\partial H$ with $\angle_{p}(c) \leqslant \theta$. Then $f$ is a decreasing function in $\theta$ and $f(\pi / 2) \leqslant R=R(\pi / 2)$. We suppose that $\angle_{p}\left(\xi_{1}, \xi_{2}\right)=\theta_{0}$. Then $\angle_{p}\left(\xi_{2}, \xi_{3}\right) \geqslant \pi-\theta_{0}$ because $\angle_{p}\left(\xi_{1}, \xi_{3}\right)=\pi$. Let $\varepsilon_{0}=(\ln 2) / R$. Since $f$ is a decreasing function in $\theta$, it is allowed that $\exp (-\varepsilon f(\theta))+$ $\exp (-\varepsilon f(\pi-\theta))$ has the minimum value $2 \exp (-\varepsilon f(\pi / 2))$. Then for all $\varepsilon<\varepsilon_{0}$, we have

$$
\begin{aligned}
\exp \left(-\varepsilon d\left(x, c_{3}\right)\right) & =\exp \left(-\varepsilon R_{0}\right) \leqslant 2 \exp \left(-\varepsilon\left(R_{0}+R\right)\right) \\
& \leqslant 2 \exp \left(-\varepsilon+f\left(\frac{\pi}{2}\right)\right) \\
& \leqslant \exp \left(-\varepsilon\left(R_{0}+f(\theta)\right)\right)+\exp \left(-\varepsilon\left(R_{0}+f(\pi-\theta)\right)\right) \\
& \leqslant \exp \left(-\varepsilon d\left(x, c_{1}\right)\right)+\exp \left(-\varepsilon d\left(x, c_{2}\right)\right) \\
& \leqslant d_{x}^{\varepsilon}\left(\xi_{1}, \xi_{2}\right)+d_{x}^{\varepsilon}\left(\xi_{2}, \xi_{3}\right) .
\end{aligned}
$$

Since the above inequality is true for any geodesic between $\xi_{1}$ and $\xi_{3}$, we have the triangle inequality

$$
d_{x}\left(\xi_{1}, \xi_{3}\right) \leqslant d_{x}\left(\xi_{1}, \xi_{2}\right)+d_{x}\left(\xi_{2}, \xi_{3}\right) .
$$

From now on, we suppose that $H$ is a visibility manifold with nonpositive sectional curvature. For convenience, we make the followings definitions.

For fixed $x \in H$ and for $\xi, \eta \in \partial H$,

$$
\begin{aligned}
l_{x}(\xi, \eta) & =\sup \left\{t \geqslant 0 \mid d\left(\gamma_{x, \xi}, \gamma_{x, \eta}\right) \leqslant d_{1}\right\} \\
\rho_{x}^{\varepsilon}(\xi, \eta) & =\exp \left(-\varepsilon l_{x}(\xi, \eta)\right) .
\end{aligned}
$$

This was suggested by Kaimanovich [5] and Hamenstädt [4], who showed that it is a metric for sufficiently small $\varepsilon$ in a negatively curved cocompact manifold. The Lemma below says that this is still a metric in $\partial H$ on the visibility manifold. 
Lemma 2.4. There exists a constant $C>0$ such that for $\xi, \eta \in \partial H$,

$$
l_{x}(\xi, \eta) \leqslant D_{x}(\xi, \eta)+C .
$$

Proof: Let $q$ be the point on a geodesic $c$ between $\xi$ and $\eta$ that is nearest to $x$. Let $\gamma_{1}$ and $\gamma_{2}$ be geodesic rays from $x$ to $\xi$ and $\eta$, respectively. Then in the triangles $(x, q, \xi)$ and $(x, q, \eta)$ the angles at the vertex $q$ equal $\pi / 2$, the distances from the point $q$ to the ray $\gamma_{1}$ and $\gamma_{2}$ is bounded by $R_{1}>0$ that is independent of the choice $\eta$ and $\xi$. Let $p_{1}, p_{2}$ be the points on the geodesic rays $\gamma_{1}, \gamma_{2}$ respectively such that $d\left(p_{i}, q\right)=d\left(q, \gamma_{i}\right)$ for $i=1,2$. Choose the points $q_{i}$ on $\gamma_{i}(i=1,2)$ such that $d\left(x, q_{i}\right)=l_{x}(\xi, \eta)$, so that $d\left(q_{1}, q_{2}\right)=d_{1}$. Let $\left[q_{1}, q_{2}\right]$ be a geodesic segment between $q_{1}$ and $q_{2}$. Then there are the three cases:

(1) $d\left(x, q_{1}\right)=d\left(x, p_{1}\right)$,

(2) $d\left(x, q_{1}\right)<d\left(x, p_{1}\right)$,

(3) $d\left(x, q_{1}\right)>d\left(x, p_{1}\right)$.

First, we consider $d\left(x, q_{1}\right)=d\left(x, p_{1}\right)$ and $d\left(x, q_{1}\right)<d\left(x, p_{1}\right)$. Then we can easily see $l_{x}(\xi, \eta) \leqslant D_{x}(\xi, \eta)$.

Second, we consider $d\left(x, q_{1}\right)>d\left(x, p_{1}\right)$. Then we can easily check $\angle_{q_{1}}(q, \xi)>\pi / 2$, because $L_{q_{1}}(q, \xi)$ is a exterior angle of the right triangle $\left(q_{1}, p_{1}, q\right)$. By the visibility axiom, the distance from $q_{1}$ to the geodesic ray $c$ from $q$ to $\xi$ is bounded above by $R_{1}>0$. Let $p$ be the point on $c$ nearest to $q_{1}$. Since $\angle_{p}\left(q, q_{1}\right)=\angle_{q}(p, x)=\pi / 2$, we can get $d(q, p) \leqslant d_{1}$, so that $d\left(x, q_{1}\right) \leqslant d(x, q)+R_{2}$ for some positive constant $R_{2}$.

Summing the above three cases, we get a constant $C>0$ so that

$$
l_{x}(\xi, \eta) \leqslant d_{x}(\xi, \eta)+C .
$$

For convenience, we use the notation $\delta$ instead of $\delta(\Gamma)$ as a critical exponent of $\Gamma$. Before the estimate of the Hausdorff measure, we prove a Lemma below, called Sullivan's Shadow Lemma in [6], which will play an important role.

Lemma 2.5. Let $\left\{\mu_{x}\right\}_{x \in M}$ be a $\delta$-conformal density of $\Gamma$. Suppose $\mu_{x}$ does not consist of a single atom. Then there are constants $C \geqslant 1$ and $b_{0} \geqslant 0$ such that for all $b \geqslant b_{0}$

$$
C^{-1} e^{-\delta d\left(x, \gamma^{-1} x\right)} \leqslant \mu_{x}\left(O_{x}\left(\gamma^{-1} x, b\right)\right) \leqslant C e^{-\delta d\left(x, \gamma^{-1} x\right)+2 b \delta} .
$$

THEOREM 2.6. Let $\left\{\mu_{x}\right\}$ be $\delta$-conformal density of $\Gamma$. There exists a constant $C>0$ such that if $A$ is a Borel subset of $L^{r}(\Gamma)$ with $\mu_{x}(A)>0$, then $\mathcal{H}_{d}^{\delta / \varepsilon}(A) \leqslant$ $C \mu_{x}(A)$, where $d=d_{x}^{\varepsilon}$.

Proof: Suppose $A \subset L^{r}(\Gamma)$ is a Borel subset. Let $x \in H$. Since $\mu_{x}(A)>0$, almost every point of $A$ is a density point of the measure in the sense that for almost 
every $a \in A$

$$
\lim _{t \rightarrow 0} \frac{\mu_{x}[B(a, t) \cap A]}{\mu_{x}[B(a, t)]}=1,
$$

where $B(a, t)=\left\{\eta \in \partial H \mid d_{x}^{\varepsilon}(a, \eta) \leqslant t\right\}$ for sufficiently small $\varepsilon>0$. Let $\Gamma=\left\{\gamma_{i}\right\}_{i=1}^{\infty}$. Let $\alpha>0$ be any constant. We can take a set $A^{\prime} \subset A$ and $t_{0}>0$, such that $\mu_{x}\left(A-A^{\prime}\right)<\alpha$ and

$$
\frac{\mu_{x}(B(a, t) \cap A)}{\mu_{x}(B(a, t))}>1-\alpha
$$

for all $0<t<t_{0}$ and all $a \in A^{\prime}$.

Let $0<b<b_{0}$, where $b_{0}$ is the constant in Lemma 2.5. Since every point in $A^{\prime} \subset L^{r}(\Gamma)$ lies in infinitely many balls $O_{x}\left(\gamma_{n}^{-1} x, b\right)$, we can construct a covering $\left\{O_{x}\left(\gamma_{n_{k}}^{-1} x, b\right)\right\}$ of $A^{\prime}$ such that $O_{x}\left(\gamma_{n_{k}}^{-1} x, b\right)$ is a ball whose $d_{x}^{\varepsilon}$-radius $r_{k}$ satisfies $r_{k}<t<t_{0} / 2$, whose centre is outside the union $\bigcup_{i=1}^{k-1} O_{x}\left(\gamma_{n_{i}}^{-1} x, b\right)$, and so that $r_{k} \geqslant r_{k+1}$ for all $k$. Then the ball with the radii $r_{k} / 2$ and the same centre are disjoint. Denote the union of these disjoint balls by $\Omega$. By Lemma 2.5, there exists $C_{1}>0$ such that

$$
\sum_{k \geqslant 1} e^{-\delta d\left(x, \gamma_{n_{k}}^{-1} x\right)} \leqslant C_{1} \mu_{x}(\Omega)
$$

By the definition of $l_{x}$ and Lemma 2.4, there exists a constant $C_{2}>0$ such that for all $k, r_{k} / 2 \leqslant C_{2} e^{-\varepsilon d\left(x, \gamma_{n_{k}}^{-1} x\right)}$. Consequently, we have

$$
\begin{aligned}
\mathcal{H}_{t}^{\delta / \varepsilon}\left(A^{\prime}\right) & \leqslant \sum_{k} r_{k}^{\delta / \varepsilon}=2^{\delta / \varepsilon} \sum_{k}\left(\frac{r_{k}}{2}\right)^{\delta / \varepsilon} \\
& \leqslant C_{3} 2^{\delta / \varepsilon} \sum e^{-\delta d\left(\gamma_{n_{k}}^{-1} x, x\right)} \\
& \leqslant C_{3} \mu_{x}(\Omega)
\end{aligned}
$$

for some constants $C_{3}>0$, and by $(2.1)$

$$
\mu_{x}(\Omega) \leqslant \frac{1}{1-\alpha} \mu_{x}(A) .
$$

Summarising these results, we have $\mathcal{H}_{t}^{\delta / \varepsilon}\left(A^{\prime}\right) \leqslant C \mu_{x}(A)$. Letting $t \rightarrow 0, \alpha$ goes to 0 . So we obtain

$$
\mathcal{H}^{\delta / \varepsilon}(A) \leqslant C \mu_{x}(A) \text {. }
$$


THEOREM 2.7. If there exists a $\delta$-conformal density $\mu_{x}$ of $\Gamma$, then the Hausdorff dimension of the radial limit set with respect to $d_{x}^{\varepsilon}$ satisfies

$$
H D\left(L^{r}(\Gamma)\right) \leqslant \frac{\delta}{\varepsilon}
$$

for sufficiently small $\varepsilon$ and for all $x \in H$.

PROOF: If $\mu_{x}\left(L^{r}(\Gamma)\right)>0$, then Theorem 2.6 implies this result. Assume $\mu_{x}\left(L^{r}(\Gamma)\right)=0$. We can get a cover of $L^{r}(\Gamma)$ as in the proof of Theorem 2.6. Then we have $\mathcal{H}_{t}^{\delta / \varepsilon}\left(L^{r}(\Gamma)\right) \leqslant C \mu_{x}(\Omega) \leqslant C \mu_{x}(\partial H)<\infty$ for some constant $C>0$.

We have an upper estimate of the Hausdorff dimension when $\Gamma$ is a just Fuchsian group. Now we prove that if a Fuchsian group $\Gamma$ is convex cocompact, $H D\left(L^{r}(\Gamma)\right)$ is exactly $\delta / \varepsilon$ with respect to the metric $d_{x}^{\varepsilon}=d$ on $\partial H$.

DEFINITION 2.8: Let $\Gamma$ be a Fuchsian group of a simply connected visibility manifold.

(i) The geodesic hull of $\Gamma$ is defined to be $G(\Gamma)=(L(\Gamma) \times L(\Gamma)-\operatorname{diag}) \times \mathbb{R}$, that is, the union of all geodesics $\gamma$ in $H$ with $\gamma(-\infty), \gamma(\infty) \in L(\Gamma) . \Gamma$ is said to be geodesic cocompact if $G(\Gamma) / \Gamma$ is compact.

(ii) The convex hull of $\Gamma$ is defined to be $H(\Gamma)$, that is, the smallest convex set in $\bar{H}$ containing $L(\Gamma) . \Gamma$ is said to be convex cocompact if $H(\Gamma) / \Gamma$ is compact.

In general $G(\Gamma)$ is not a convex set, but $G(\Gamma)$ is a subset of $H(\Gamma)$.

THEOREM 2.9. The followings are equivalent.

(1) $\Gamma$ is convex cocompact.

(2) $\Gamma$ is geodesic cocompact.

(3) For any point $\xi \in \partial H$, there is a constant $C>0$ such that the geodesic ray $\gamma$ from $x$ to $\xi$ is in the $C$-neighbourhood of a orbit of $\Gamma$.

Proof: To show (1) implies (2), we first suppose that $g(\Gamma) / \Gamma$ is compact. Since $G(\Gamma)$ is a subset of $H(\Gamma), H(\Gamma) / \Gamma$ is compact.

To show (2) implies (1), we first have by the visibility axiom, that for any $\xi_{1}, \xi_{2}, \xi_{3} \in \bar{H}$, every point of the interior part of a triangle $\left(\xi_{1}, \xi_{2}, \xi_{3}\right)$ is in the $R(\pi / 2)$ neighbourhood of the triangle, where $R(\pi / 2)$ is a constant corresponding to $\pi / 2$ in the uniform visibility axiom. Further, we get that $H(\Gamma)$ is in the $R(\pi / 2)$-neighbourhood of the $G(\Gamma)$. So we get that the geodesic cocompactness of $\Gamma$ implies the convex cocompact of $\Gamma$.

To show (3) implies (2), we first choose any two distinct points $\xi$ and $\eta$ in $L(\Gamma)$. Let $\gamma_{x \xi}$ and $\gamma_{x \eta}$ be the geodesic rays from $x$ to $\xi$ and $\eta$, respectively and let $\gamma_{\xi \eta}$ be 
the geodesic line from $\xi$ to $\eta$. Then by the uniform visibility axiom, $\gamma_{x \xi}$ and $\gamma_{x \eta}$ are in the $R(\pi / 2)$-neighbourhood of $\gamma_{\xi \eta}$. Since $\gamma_{x \xi}$ and $\gamma_{x \eta}$ are in the $C$-neighbourhood of a orbit of $\Gamma, \gamma_{\xi \eta}$ is in the $(C+R(\pi / 2))$-neighbourhood of a orbit of $\Gamma$, and $\Gamma$ is geodesic cocompact.

To show (2) implies (3), we suppose $\Gamma$ is geodesic cocompact. Then there is a constant $C_{1}>0$ such that any point of $G(\Gamma)$ has a distance less than $C_{1}$ from an orbit of $\Gamma$. Let $\xi \in \partial H$ be any point. Let $x \in H$ be a fixed point. Choose a distinct point $\eta \in \partial H$ from $\xi$. We can get a unique point $p$ in a geodesic $\gamma_{\xi \eta}$ from $\xi$ to $\eta$ such that $d(x, p)=d\left(x, \gamma_{\xi \eta}\right)$. Let $d(x, p)=C_{2}$. It is easy to see that $\gamma_{x \xi}$ is in the $R(\pi / 2)$-neighbourhood of $\gamma_{p \xi}$ and $\gamma_{x p}$. By the compactness of $G(\Gamma) / \Gamma, \gamma_{p \xi}$ is in the $C_{1}$-neighbourhood of a orbit of $\Gamma$. And there exists a constant $C_{3}>0$ such that the ball with centre $x$ and the radius $R(\pi / 2)$ is in the $C_{3}$ neighbourhood of a orbit of $\Gamma$. Let $C=\min \left\{C_{1}+C_{2}, C_{3}\right\}$. Therefore the geodesic ray $\gamma_{x \xi}$ is in the $C$-neighbourhood of a orbit of $\Gamma$.

Theorem 2.9 says that every limit point in the convex cocompact set is a radial limit point and $L(\Gamma)=L^{r}(\Gamma)$.

Lemma 2.10. Suppose $\Gamma$ is a convex cocompact group. Let $\mu_{x}$ be a $\delta$-conformal density. Then there exists $C>0$ and $r_{0}>0$ such that

$$
\mu_{x}(B(\xi, r)) \leqslant C r^{\delta / \varepsilon}
$$

where $B(\xi, r)=\left\{\eta \in \partial H \mid d_{x}^{\varepsilon}(\xi, \eta)<r\right\}$ is a ball with centre $\xi \in L(\Gamma)$ and radius $r_{0}>r>0$.

Proof: By Theorem 2.8, there is a $C_{1}>0$ such that any point on a geodesic ray from $x \in H$ has a point of the orbit of $\Gamma$ in a distance $C_{1}>0$. Consider $\xi \in L(\Gamma)$ and $e^{-\varepsilon R}>r>0$ where $R=R(\pi / 2)$ is the constant corresponding to the angle $\pi / 2$ in the definition of the uniform visibility axiom. Choose a point $u$ on the geodesic ray from $x$ to $\xi$ such that $d(x, u)=-\ln r / \varepsilon$. We can get a point $\alpha^{-1}(x)$ of orbit $\Gamma(x)$ such that $d\left(u, \alpha^{-1}(x)\right) \leqslant C_{1}$ for some $\alpha \in \Gamma$. Let $\eta$ be a point in $B(\xi, r)$. Let $p \in H$ be the point on the geodesic line $\gamma_{\xi \eta}$ between $\xi$ and $\eta$ such that $d(x, p)=d\left(x, \gamma_{\xi \eta}\right)$. Then we have that both of $\angle_{p}(\xi, x)$ and $\angle_{p}(\eta, x)$ are $\pi / 2$, By the definition of uniform visibility, we get points $q_{1}$ and $q_{2}$ on the geodesic rays $\gamma_{x \xi}$ and $\gamma_{x \eta}$, respectively, such that both of $d\left(p, q_{1}\right)$ and $d\left(p, q_{2}\right)$ are less than and equal to $R(\pi / 2)$. By the triangle inequality, we have $d\left(q_{1}, q_{2}\right) \leqslant 2 R$, and by the angle comparison of triangle, we have $d\left(u, q_{1}\right) \leqslant R$. Since

$$
d\left(\alpha^{-1}(x), \gamma_{x \eta}\right) \leqslant d\left(\alpha^{-1}(x), u\right)+d\left(u, q_{1}\right)+d\left(q_{1}, q_{2}\right) \leqslant C_{1}+3 R,
$$

we have $\eta \in O_{x}\left(\alpha^{-1}(x), C_{1}+3 R\right)$. So it means that $B(\xi, r) \subset O_{x}\left(\alpha^{-1}(x), C_{1}+3 R\right)$. 
By Lemma 2.5, we have

$$
\begin{aligned}
\mu_{x}(B(\xi, r)) & \leqslant \mu_{x}\left(O_{x}\left(\alpha^{-1}(x), C_{1}+3 R+d_{1}\right)\right) \\
& \leqslant C_{2} e^{-\delta d\left(x, \alpha^{-1}(x)\right)} .
\end{aligned}
$$

where $C_{2}>0$ is a constant independent of $x$ and $r$. Since $d\left(x, \alpha^{-1}(x)\right)>-\ln r / \varepsilon-$ $C_{1}>0$, we have a constant $C>0$ such that

$$
\mu_{x}(B(\xi, r)) \leqslant C r^{\delta / \varepsilon}
$$

THEOREM 2.11. Let $\Gamma$ be a convex cocompact group with critical exponent $\delta=\delta(\Gamma)$. Then there exists a constant $C>0$ such that if $A$ is a Borel subset of the limit set then we have

$$
\mu_{x}(A) \leqslant C \mathcal{H}_{\mathrm{d}}^{\delta / \varepsilon}(A)
$$

Proof: Let $\left\{B\left(\xi_{i}, r_{i}\right)\right\}$ be any cover of $A$ where the balls $B\left(\xi_{i}, r_{i}\right)$ in $\partial H$ have centres on the linit set. Let $\varepsilon>0$. Then by Theorem 2.10, we get for $\varepsilon>r_{i}>0$,

$$
\mu_{x}(A) \leqslant \sum_{i} \mu_{x}\left(B\left(\xi_{i}, r_{i}\right)\right) \leqslant C \sum_{i} r_{i}^{\delta / \epsilon}
$$

Letting $\varepsilon \rightarrow 0$, we have a constant $C>0$ such that

$$
\mu_{x}(A) \leqslant C \mathcal{H}_{d}^{\delta / \varepsilon}(A)
$$

THEOREM 2.12. If $\Gamma$ is a convex cocompact group with critical exponent $\delta$ then the Hausdorff dimension of the limit set is $\delta / \varepsilon$ with respect to the metric $d_{x}^{\varepsilon}$.

Proof: By Theorem 2.9, we have $L^{r}(\Gamma)=L(\Gamma)$. Since $\mu_{x}\left(L^{r}(\Gamma)\right)=\mu_{x}(L(\Gamma))$ has the positive full measure, Theorem 2.6 implies $H D(L(\Gamma)) \leqslant \delta / \varepsilon$. By Theorem 2.11, we have $H D(L(\Gamma)) \geqslant \delta / \varepsilon$, which means that the Hausdorff dimension of the limit set is $\delta / \varepsilon$ exactly.

\section{REFERENCES}

[1] W. Ballmann, M. Gromov and V. Schroeder, Manifolds of nonpositive curvature, Progress in Mathematics 61 (Birkhauser Boston Inc, Boston MA, 1985).

[2] P. Eberlein, 'Geodesic flow in certain manifolds without conjugate points', Trans. Amer. Math. Soc. 167 (1972), 151-170.

[3] P. Eberlein and B. O'Neil, 'Visibility manifolds', Pacific J. Math. 46 (1973), 45-109. 
[4] U. Hamenstädt, 'A new description of the Bowen-Margulis measure', Ergodic Theory Dynamical Systems 9 (1989), 455-464.

[5] V.A. Kaimanovich, 'Invariant measure for the geodesic flow and measures at infinity on negatively curved manifolds', Ann. Inst. H. Poincaré Phys. Théor. 53 (1990), 361-393.

[6] V.H. Kim, 'Conformal density of visibility manifold', Bull. Korean Math. Soc. 38 (2001), 211-222.

[7] S.J. Patterson, Lectures on measures on limit sets of Kleinian group, London Math. Soc. Lecture Note Ser. 111 (Cambridge Univesity Press, Cambridge, 1987), pp. 281-323.

[8] D. Sullivan, 'The density at infinity of a discrete group of hyperbolic motions', Inst. Hautes Études Sci. Publ. Math. 50 (1979), 171-202.

[9] C. Yue, 'The ergodic theory of discrete isometry groups on manifolds of variable negative curvature', Trans. Amer. Math. Soc. 348 (1996), 4965-5005.

\author{
Department of Mathematics \\ Hoseo University \\ Baebang Myun \\ Asan 337-795 \\ Korea \\ e-mail: hjkim @math.hoseo.ac.kr
}

